\title{
Unchanged triclabendazole kinetics after co- administration with ivermectin and methimazole: failure of its therapeutic activity against triclabendazole-resistant liver flukes
}

\author{
Laura Ceballos ${ }^{1,2+}$, Laura Moreno ${ }^{1,2 \dagger}$, Luis Alvarez ${ }^{1,2}$, Laura Shaw ${ }^{3}$, lan Fairweather ${ }^{3}$, Carlos Lanusse L $^{1,2^{*}}$
}

\begin{abstract}
Background: The reduced drug accumulation based on enhanced drug efflux and metabolic capacity, identified in triclabendazole (TCBZ)-resistant Fasciola hepatica may contribute to the development of resistance to TCBZ. The aim of this work was to evaluate the pharmacokinetics and clinical efficacy of TCBZ administered alone or coadministered with ivermectin (IVM, efflux modulator) and methimazole (MTZ, metabolic inhibitor) in TCBZ-resistant F. hepatica-parasitized sheep. Sheep infected with TCBZ-resistant F. hepatica (Sligo isolate) were divided into three groups $(n=4)$ : untreated control, TCBZ-treated (i.r. at $10 \mathrm{mg} / \mathrm{kg}$ ) and TCBZ+IVM+MTZ treated sheep (10 i.r., 0.2 s.C. and $1.5 \mathrm{i.m.} \mathrm{mg} / \mathrm{kg}$, respectively). Plasma samples were collected and analysed by HPLC. In the clinical efficacy study, the animals were sacrificed at 15 days post-treatment to evaluate the comparative efficacy against TCBZresistant F. hepatica.
\end{abstract}

Results: The presence of IVM and MTZ did not affect the plasma disposition kinetics of TCBZ metabolites after the i.r. administration of TCBZ. The AUC value of TCBZ.SO obtained after TCBZ administration $(653.9 \pm 140.6 \mu \mathrm{g} . \mathrm{h} / \mathrm{ml})$ was similar to that obtained after TCBZ co-administered with IVM and MTZ $(650.7 \pm 122.8 \mu \mathrm{g} . \mathrm{h} / \mathrm{ml})$. Efficacy values of 56 and 38\% were observed for TCBZ alone and for the combined treatment, respectively. No statistical differences $(P>0.05)$ were observed in fluke counts between treated groups and untreated control, which confirm the resistant status of the Sligo isolate.

Conclusions: The presence of IVM and MTZ did not affect the disposition kinetics of TCBZ and its metabolites. Thus, the combined drug treatment did not reverse the poor efficacy of TCBZ against TCBZ-resistant $F$. hepatica.

\section{Background}

Triclabendazole (TCBZ, 6-chloro-5(2-3 dichlorophenoxy)-2-methyl thio-benzimidazole), an halogenated benzimidazole (BZD) thiol derivative, shows high efficacy against both the immature and mature stages of Fasciola hepatica in sheep and cattle, which is a differential feature compared to other available trematodicidal drugs [1]. As a consequence of its excellent activity against the liver fluke, it has been extensively used and this has inevitably promoted the selection of TCBZ-

\footnotetext{
* Correspondence: clanusse@vet.unicen.edu.ar

+ Contributed equally

'Laboratorio de Farmacología, Facultad de Ciencias Veterinarias, Universidad Nacional del Centro de la Provincia de Buenos Aires (UNCPBA), Campus Universitario, 7000, Tandil, Argentina
}

(c) 2010 Ceballos et al; licensee BioMed Central Ltd. This is an Open Access article distributed under the terms of the Creative Commons Attribution License (http://creativecommons.org/licenses/by/2.0), which permits unrestricted use, distribution, and reproduction in any medium, provided the original work is properly cited. resistant populations, which is now a worrying problem in several areas of the world $[2,3]$.

Parasites have several possible strategies to achieve drug resistance, including changes in the target molecule, in drug uptake/efflux mechanisms and in drug metabolism [4]. At least two mechanisms appear to be implicated in TCBZ resistance in F. hepatica: increased drug efflux and enhanced oxidative metabolism [5-7]. TCBZ and its sulphoxide metabolite (TCBZ.SO) are both substrates of P-glycoprotein (Pgp) [8]. Over-expression of Pgp has been implicated in the resistance to macrocyclic lactones (ivermectin (IVM), moxidectin $(\mathrm{MXD}))[9,10]$, closantel and BZDs in nematodes [11]), although the exact nature of the role has yet to be established [12]. Different ex vivo experiments support 
the hypothesis of the involvement of Pgp over-expression in the resistance of $F$. hepatica to TCBZ. Higher levels of TCBZ and TCBZ.SO were observed within TCBZ-resistant flukes when drug efflux from the parasite was decreased by IVM [7], a well recognized Pgp substrate/inhibitor $[9,13]$. It has been demonstrated that TCBZ and its main metabolites, TCBZ.SO and TCBZsulphone $\left(\mathrm{TCBZ} \mathrm{SO}_{2}\right)$ may induce tegumental damage in liver flukes [14]. Additionally, an increased oxidative metabolic capacity has been described as complementary TCBZ resistance mechanism in F. hepatica $[5,6]$. In fact, co-incubation of TCBZ or TCBZ.SO with methimazole (MTZ), a flavin monooxygenase (FMO) enzymatic system inhibitor, lead to more severe surface morphological changes in TCBZ-resistant $F$. hepatica, compared to that observed after incubation with TCBZ or TCBZ.SO alone [15].

The interaction between co-administered drugs may induce changes in the pharmacokinetic behaviour of either molecule. Increased albendazole sulphoxide plasma concentrations in lambs after co-administration of albendazole (intraruminally, i.r.) with IVM (subcutaneously, s.c.), was previously reported [16]. Similarly, after the co-administration to sheep of IVM and TCBZ by the intravenous (i.v.) route, an enhanced TCBZ.SO plasma concentration was achieved [17]. On the other hand, MTZ inhibition of TCBZ oxidative metabolism by sheep liver microsomes has been reported [18]. However, MTZ did not affect TCBZ disposition kinetics in sheep after the administration of both compounds by the i.v. route [19].

Both modified influx/efflux and enhanced metabolism may account for the development of resistance to TCBZ in F. hepatica. As a consequence, it opens up the possibility of modulating drug efflux and metabolism in the TCBZ-resistant fluke, by co-administering TCBZ with MTZ and IVM, with the aim of reversing anthelmintic resistance. Furthermore, in vivo drug-drug interaction between these drugs may modify the overall disposition kinetics and pattern of drug distribution of TCBZ to the liver fluke. The aims of the current work were: a) to investigate the potential effect of MTZ and IVM on the plasma concentrations profiles of TCBZ and its metabolites in sheep and b) to study the clinical efficacy of TCBZ alone or when co-administered with MTZ and IVM against TCBZ-resistant $F$. hepatica.

\section{Methods}

\section{Chemicals}

Pure reference standards (97-99\%) of TCBZ and its TCBZ.SO and TCBZ.SO $\mathrm{S}_{2}$ metabolites, were provided by Novartis Animal Health (Basel, Switzerland) (Batch \# AMS 215/102, HI-1025/1 and JG-5161/6, respectively). MTZ and IVM were purchased from Sigma-Aldrich
Chemical Company (St Louis, USA). The different solvents (HPLC grade) and buffer salt used for sample extraction or chromatographic methods were purchased from Baker Ind. (Phillipsburg, USA).

\section{Animals and Experimental design}

Twelve (12) healthy intact male Corriedale sheep (53.8 \pm $2.6 \mathrm{~kg}$ ) aged 14-16 months and obtained from a farm located in an area free of $F$. hepatica were involved in this trial. Additionally, the absence of liver fluke infection was checked by analysis of F. hepatica eggs in faeces, following routine procedures [20]. Animals were housed individually during the experiment and for 20 days before the start of the study. Animals were fed on a commercial balanced concentrate diet. Water was provided ad libitum. Animal procedures and management protocols were carried out in accordance with the Animal Welfare Policy (Act 087/02) of the Faculty of Veterinary Medicine, Universidad Nacional del Centro de la Provincia de Buenos Aires (UNCPBA), Tandil, Argentina http://www.vet.unicen.edu.ar and internationally accepted animal welfare guidelines [21].

Animals were each orally infected with eighty (80) metacercariae of a TCBZ-resistant $F$. hepatica isolate, named Sligo. For details of the history of the Sligo isolate, see previous works $[5,22,23]$. Sixteen weeks after infection, animals were randomly distributed into three experimental groups $(\mathrm{n}=4$ each): Group I, which represented the untreated control group; Group II, in which animals were treated with TCBZ (Fasinex ${ }^{\oplus}$, Novartis) by the i.r. route at $10 \mathrm{mg} / \mathrm{kg}$ dose rate; and Group III, in which animals were simultaneously treated with TCBZ (Fasinex ${ }^{\oplus}$, Novartis) by the i.r. route $(10 \mathrm{mg} / \mathrm{kg})$ and IVM (Ivosint ${ }^{\circ}$, Biogénesis) by the s.c. route $(0.2 \mathrm{mg} / \mathrm{kg}$, internal face of the thigh). Additionally, animals in Group III were treated by the intramuscular (i.m.) route (Semitendinosus muscle) with MTZ (2.5\% aqueous solution) at a dose rate of $1.5 \mathrm{mg} / \mathrm{kg}$ [24]. MTZ administration was performed $30 \mathrm{~min}$ after TCBZ/IVM treatment. Blood samples $(5 \mathrm{ml})$ were taken by jugular venipunctures into heparinised Vacutainers ${ }^{\bullet}$ tubes (Becton Dickinson, USA) before administration (time 0 ) and at 1,3 , $6,9,12,18,24,30,36,48,72,96,120$ and 144 h posttreatment. Plasma was separated by centrifugation at $3000 \mathrm{~g}$ for $15 \mathrm{~min}$, placed into plastic tubes and frozen at $-20^{\circ} \mathrm{C}$ until analyzed by high performance liquid chromatography (HPLC).

\section{Clinical efficacy study}

Fifteen days after treatment all animals were stunned and exsanguinated immediately. Adult $F$. hepatica specimens were recovered from the common bile ducts and the gall bladder of each sheep and counted according to the World Association for the Advancement of 
Veterinary Parasitology (W.A.A.V.P) guidelines [25]. The efficacy of each anthelmintic treatment was determined by the comparison of $F$. hepatica burdens in treated versus untreated animals. The following equation expresses the percent efficacy $(\% E)$ of a drug treatment against $F$. hepatica (F.h.) in a single treatment group (T) when compared with an untreated control $(C)$.

$\% E=($ Mean of F.h. in $C-$ Mean of F.h. in $T) /($ Mean of F.h. in $C) * 100$

The geometric mean was used as it most accurately represents the distribution of parasite populations within each group [25].

\section{Analytical procedures \\ Plasma sample extraction}

TCBZ and its metabolites were extracted from plasma as previously described [18]. Samples $(1 \mathrm{ml})$ were spiked with $10 \mu \mathrm{l}$ of oxibendazole (OBZ) $(100 \mu \mathrm{g} / \mathrm{ml})$, used as internal standard. After addition of $2 \mathrm{ml}$ of acetonitrile, samples were shaken for $20 \mathrm{~min}$ (multivortex) and then centrifuged at $2500 \mathrm{~g}$ for $15 \mathrm{~min}$. The supernatants were recovered and evaporated to dryness in a vacuum concentrator (Speed-Vac ${ }^{\oplus}$, Savant, Los Angeles, USA). The dry extracts were reconstituted in $300 \mu \mathrm{l}$ of mobile phase and an aliquot of $50 \mu \mathrm{l}$ was injected into the HPLC system.

\section{Drug quantification by HPLC analysis}

Experimental and fortified plasma samples were analysed by HPLC to determine the concentration of TCBZ and its metabolites following the methodology previously described [18]. The elution from the stationary phase (Selectosil $\mathrm{C}_{18}$ column, $5 \mu \mathrm{m}, 250 \times 4.6$ $\mathrm{mm}$, Phenomenex ${ }^{\circ}, \mathrm{CA}$, USA) was carried out at a flow rate of $1.2 \mathrm{ml} / \mathrm{min}$, using a mixture of acetonitrile/ammonium acetate $(0.025 \mathrm{M}, \mathrm{pH}$ 6.6) as mobile phase. Fifty $\mu \mathrm{l}$ of each previously extracted sample were injected into a Shimadzu 10 A HPLC System (Kyoto, Japan), using a gradient pump, UV detector set at $300 \mathrm{~nm}$, an autosampler and a controller (Shimadzu Class LC10, Kyoto, Japan). Analytes were identified by the retention times of pure reference standards. Chromatographic retention times were: 4.09 (OBZ), 5.91 (TCBZ.SO), 7.95 (TCBZ.SO ${ }_{2}$ ) and 10.36 (TCBZ) min. Calibration curves for each analyte were prepared by least squares linear regression analysis, which showed correlation coefficients between 0.995 and 0.998 . The absolute recovery of drug analytes from plasma was calculated by comparison of the peak areas from spiked plasma samples with the peak areas resulting from direct injections of standards in mobile phase. Mean absolute recoveries and coefficient of variations $(\mathrm{CV})$ within the concentration range between 0.1 and $25 \mu \mathrm{g} / \mathrm{ml}$ (triplicate determinations) were $89.2 \%$ (CV:
6.74\%) (TCBZ), 87.1\% (CV: 6.03\%) (TCBZ.SO) and 90.1\% (CV: 4.75\%) (TCBZ.SO ${ }_{2}$ ). Precision (intra- and inter-assay) was determined by analysing replicates of fortified plasma samples $(n=5)$ with each compound at three different concentrations $(0.1,5$ and $10 \mu \mathrm{g} / \mathrm{ml})$. $\mathrm{CV}$ ranged from 2.54 to $14.6 \%$. The limit of quantification (LOQ) was defined as the lowest measured concentration with a CV $<20 \%$ and accuracy of $\pm 20 \%$ and an absolute recovery $\geq 70 \%$. The LOQ defined for the three molecules assayed was $0.1 \mu \mathrm{g} / \mathrm{ml}$. Values below LOQ were not included in the pharmacokinetic analysis.

\section{Pharmacokinetic analysis}

The concentration versus time curves for TCBZ metabolites in plasma for individual animals were fitted with the PKSolutions ${ }^{\text {Tw }}$ computer program/programme (Summit Research Service, Ashland, USA). Pharmacokinetic analysis of the experimental data was performed by non-compartmental analysis. The first order absorption rate constant $\left(k_{a b}\right)$ or the first order metabolite formation rate constant $\left(\mathrm{k}_{\mathrm{f}}\right)\left(\mathrm{h}^{-1}\right)$ were calculated by the residual method [26]. The elimination half life (T1/2el) and absorption (T1/2ab) or metabolite formation (T1/2for) half lives were calculated as $\ln 2 / \beta$ and $\ln 2 / \mathrm{k}$, respectively, where $\beta$ represent the terminal slope $\left(h^{-1}\right)$. The observed peak concentration $\left(\mathrm{C}_{\max }\right)$ and time to peak concentration $\left(\mathrm{T}_{\max }\right)$ were read from the plotted concentration-time curve of each analyte. The area under the concentration time-curve (AUC) was calculated by the trapezoidal rule [26] and further extrapolated to infinity by dividing the last experimental concentration by the terminal slope $(\beta)$.Statistical moment theory was applied to calculate the mean residence time (MRT) for metabolites in plasma, as follows:

$$
\text { MRT }=\text { AUMC } / \text { AUC }
$$

where AUC is as defined previously and AUMC is the area under the curve of the product of time and the plasma drug concentration versus time from zero to infinity [26].

\section{Statistical analysis of the data}

Pharmacokinetic parameters are presented as mean \pm SD. The Student' t-test was used for the statistical comparison of the pharmacokinetic data obtained from both treatments groups. AUC, Cmax, MRT, T1/2el and T1/ 2 for values were log-transformed before statistical analysis. Untransformed Tmax values were compared by non-parametric test (Mann-Withney Test). Fluke counts in each experimental group were compared by non parametric test (Kruskal-Wallis test). In all cases a value of $P<0.05$ was considered statistically significant. 


\section{Results}

TCBZ.SO and TCBZ.SO $\mathrm{S}_{2}$ were the only analytes recovered in plasma after the i.r. administration of TCBZ alone or co-administered with IVM and MTZ in TCBZresistant $F$. hepatica-infected sheep. High concentrations of both TCBZ metabolites were measured in plasma up to $144 \mathrm{~h}$ post-treatment (in both treated groups). The comparative mean $( \pm \mathrm{SD})$ plasma concentration profiles of TCBZ.SO and TCBZ.SO $\mathrm{S}_{2}$ obtained after the i.r. administration of TCBZ alone or co-administered with IVM and MTZ are shown in Figure 1 (TCBZ.SO) and Figure $2\left(\mathrm{TCBZ}_{\mathrm{SO}}\right)$. Table 1 summarizes the plasma pharmacokinetic parameters obtained for TCBZ.SO and TCBZ. $\mathrm{SO}_{2}$ after the i.r. administration of TCBZ alone or co-administered with IVM and MTZ to F. hepaticainfected sheep. The presence of IVM and MTZ did not affect the plasma disposition kinetics of TCBZ metabolites after the i.r. administration of TCBZ. The AUC value obtained for TCBZ.SO after TCBZ administration $(653.9 \pm 140.6 \mu \mathrm{g} . \mathrm{h} / \mathrm{ml})$ was similar to that obtained after TCBZ co-administered with IVM and MTZ (650.7 $\pm 122.8 \mu \mathrm{g} . \mathrm{h} / \mathrm{ml}$ ).

Table 2 shows parasite counts and the clinical efficacy (\%) of TCBZ against TCBZ-resistant $F$. hepatica, after its i.r. administration alone or co-administered with IVM and MTZ. Efficacy values of 56 and 38\% were observed for TCBZ alone and for the combined treatment, respectively. No statistical differences $(\mathrm{P}>0.05)$ were observed in fluke counts between treated groups and untreated control, which confirm the resistant status of the Sligo isolate.

\section{Discussion}

The use of drug combinations is becoming an alternative tool for therapeutic control of anthelmintic-resistant parasites. However, it is important to understand the potential pharmacokinetic/pharmacodynamic interactions between anthelmintic molecules, before drug combination formulations are developed to be introduced onto the pharmaceutical market.

Consistent with kinetic data previously obtained in sheep [27], TCBZ.SO and TCBZ.SO ${ }_{2}$ were the main metabolites recovered in plasma after the i.r. administration of TCBZ, which has been related to a first-pass oxidation occurring mainly in the liver. TCBZ.SO accounted for $42 \%$ of the total analytes found in sheep plasma after the i.r. administration of TCBZ. Both TCBZ metabolites were recovered in plasma for a period of $144 \mathrm{~h}$ post-treatment. The long persistence and high concentrations of TCBZ.SO and TCBZ.SO $\mathrm{S}_{2}$ in sheep plasma compared with other BZD anthelmintics [28] are explained by the strong binding of both metabolites to plasma proteins [27]. This pharmacological property offers some advantage to TCBZ compared to other benzimidazole compounds in the activity against blood-feeding adult flukes.

Sulphoxidation and sulphonation are the main metabolic reactions involved in TCBZ hepatic biotransformation in sheep. A recent in vitro work showed that both mixed function oxidases, FMO and cytochrome P-450 (CYP), are involved in such metabolic reactions in sheep liver [18]. It was demonstrated that TCBZ sulphoxidative metabolism was reduced in the presence of the FMO inhibitor MTZ and also when the anthelmintic molecule was incubated in the presence of the CYP inhibitor piperonyl butoxide (PB) [18]. On the other hand, in vivo interference with the liver FMO-mediated and/or CYP-mediated metabolism has been shown to result in pronounced modifications of the pharmacokinetic behaviour of anthelmintically active BZDs metabolites. For example, in sheep, co-administration of oxfendazole with MTZ [29] increased the concentration of the active moieties (fenbendazole and oxfendazole) in the systemic circulation. Furthermore, MTZ and metyrapone, a potent inhibitor of the CYP system, improved the plasma availabilities of albendazole metabolites following the administration of the pro-BZD netobimin to sheep [30].

The pharmacokinetic interaction between albendazole and IVM in sheep was recently demonstrated. Higher albendazole sulphoxide plasma AUC was obtained after the i.r. administration of $\mathrm{ABZ}$ co-administered with IVM given s.c. to lambs [16]. Moreover, the co-administration of TCBZ and IVM by the i.v. route to sheep was correlated with higher peak plasma concentrations of TCBZ metabolites compared to those obtained following TCBZ alone [17]. The mechanism implicated in TCBZIVM interaction remains unclear. However, since both compounds have been described as Pgp substrates $[8,9,14]$, it could be based on a drug-drug interaction via a transporter(s)-mediated drug efflux mechanism. Additionally, considering that IVM [31] as well as TCBZ and its metabolites [27] are strongly bound to plasma proteins, a drug binding displacement may occur when TCBZ and IVM are co-administered. Any interaction between IVM and MTZ has been described in the literature.

When TCBZ was administered by the i.r. route simultaneously with IVM (s.c.) and MTZ (i.m.) (current experiment), the plasma concentration profile of TCBZ. $\mathrm{SO}$ and TCBZ.SO $\mathrm{S}_{2}$ were similar to that observed after the administration of TCBZ alone. In agreement with our results, the presence of MTZ did not modify the plasma pharmacokinetic behaviour of TCBZ metabolites in sheep [19], which indicates that the interaction between TCBZ and MTZ observed under in vitro conditions is not achieved in vivo. Furthermore, while IVM 


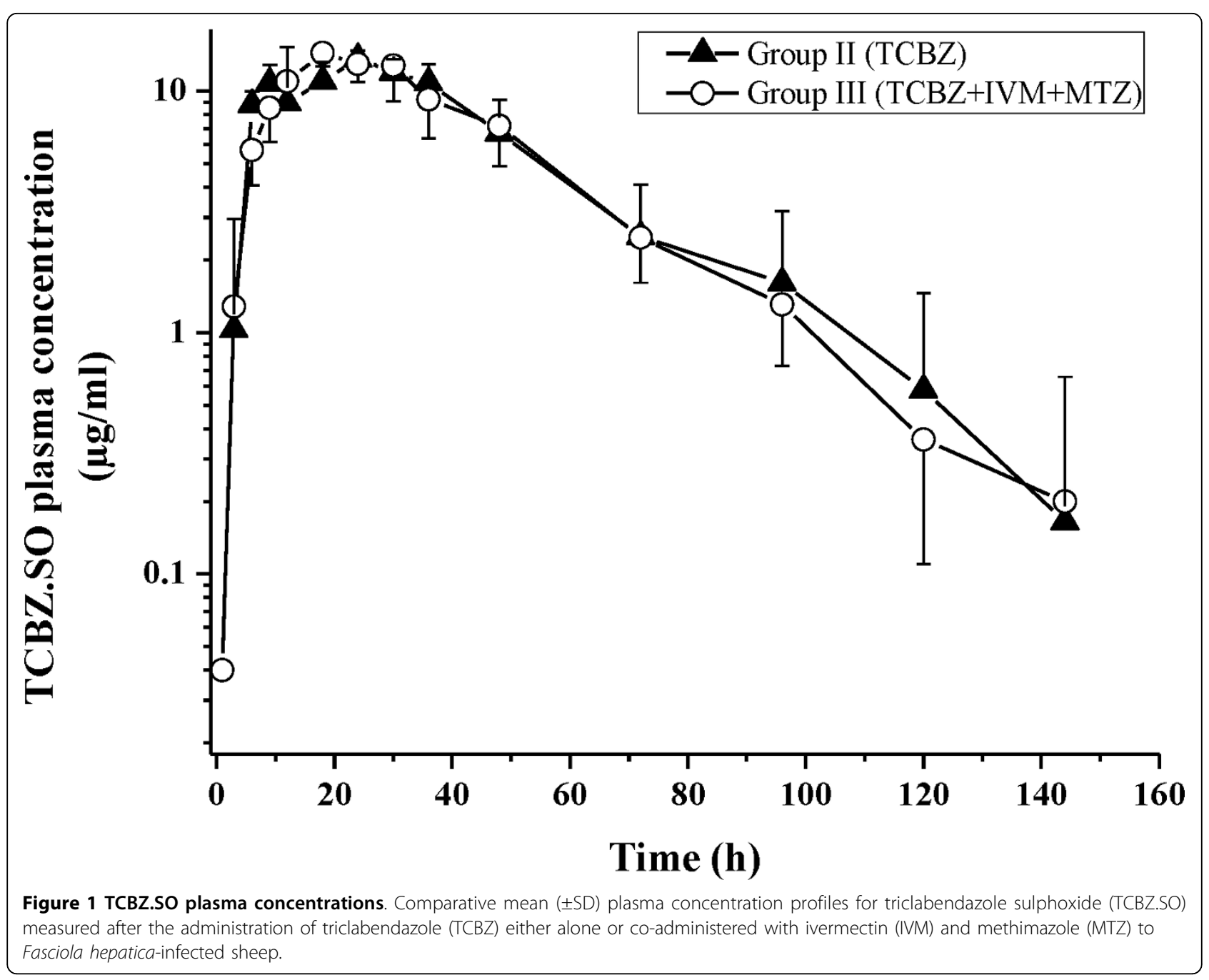

modifies the plasma pharmacokinetic behaviour of TCBZ metabolites after its simultaneous i.v. administration [17], the presence of IVM (after its s.c. administration) did not affect the plasma pharmacokinetic behaviour of TCBZ metabolites (after the i.r. administration of TCBZ). However, when we analyze the differences in IVM plasma concentrations according to the route of administration, we can observe that after its i.v. administration, IVM reaches an initial plasma concentration of $281.2 \pm 32.6 \mathrm{ng} / \mathrm{ml}$, which was significantly higher than its peak plasma concentration $(21.3 \pm 13.3$ $\mathrm{ng} / \mathrm{ml}$ ) obtained after the s.c. administration at the same dose rate (Alvarez et al., 2008). It is clear that the route of administration may influence the drug concentration profiles over time at the different tissue/fluids. Thus, the potential drug-drug interaction may be influenced by the used route of administration of each compound

The WAAVP guidelines indicate that drug efficacy should be expressed as highly effective (over 98\%), effective (90-98\%), moderately effective (80-89\%) or insufficiently active (less than 80\%) [25]. According to this guideline, the results obtained in the present trial confirm the high level of resistance to TCBZ for the Sligo isolate, which has been described previously $[22,23,32]$. The experimental evidence, accumulated after different ex vivo experiments [5-7,15], demonstrate that altered drug efflux and enhanced metabolism may contribute to the development of resistance to TCBZ in F. hepatica. The co-incubation of TCBZ or TCBZ.SO with IVM results in higher drug accumulation into TCBZ-resistant F. hepatica compared to that observed after the incubation of TCBZ or TCBZ.SO alone [7]. Furthermore, the rate of sulphoxidative metabolism of TCBZ into TCBZ.SO was significantly higher $(>40 \%)$ in TCBZ-resistant flukes [6]. This metabolic pathway described in the resistant flukes was significantly inhibited by MTZ [6]. From the results obtained in the present work, we can conclude that the co-administration of TCBZ with a Pgp substrate/inhibitor (IVM) and a metabolic inhibitor (MTZ) did not increase the clinical 


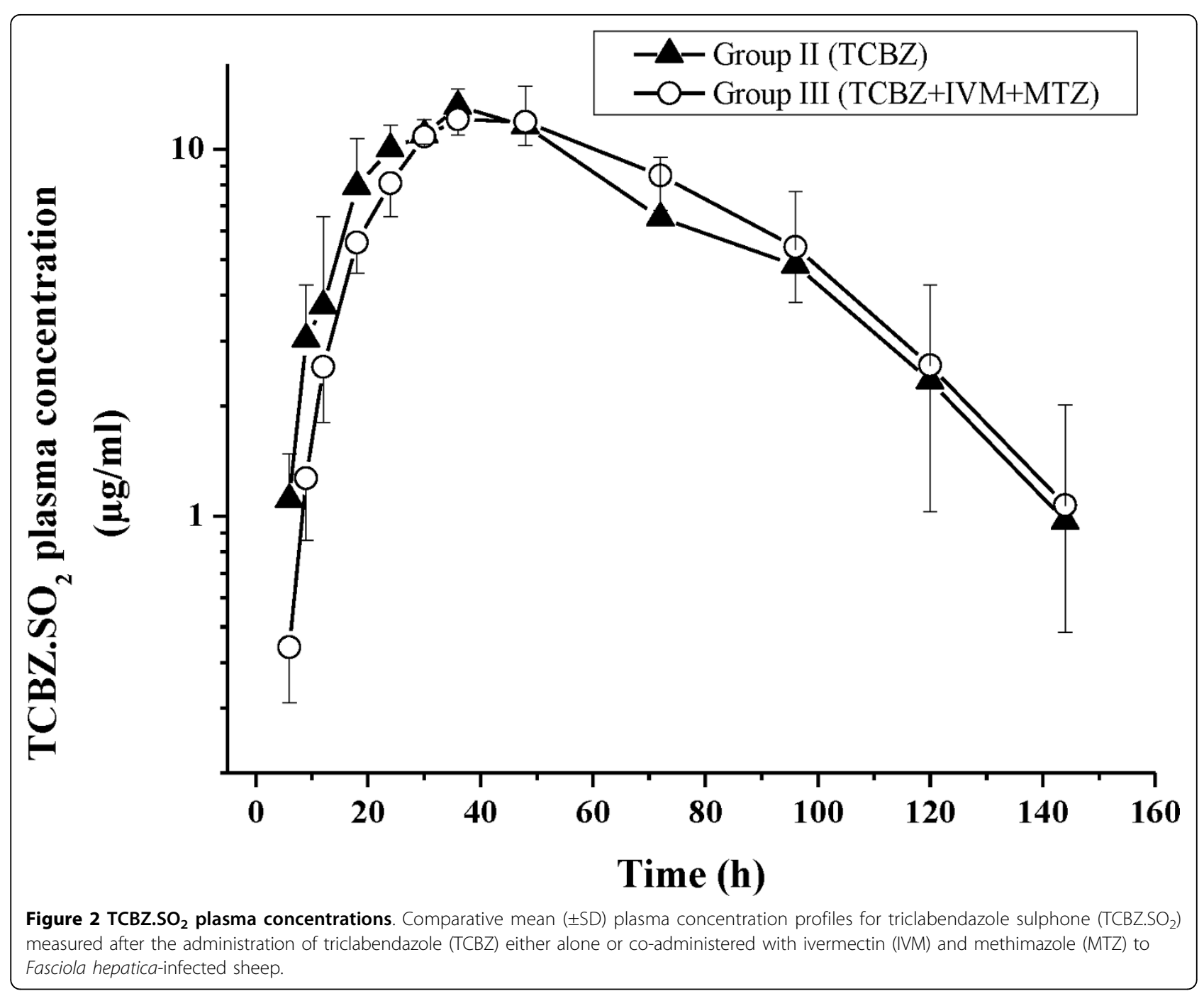

Table 1 Plasma pharmacokinetic parameters (mean \pm SD) for triclabendazole sulphoxide (TCBZ.SO) and triclabendazole sulphone (TCBZ.SO ${ }_{2}$ ) obtained after the intraruminal (i.r.) administration of triclabendazole (TCBZ, 10 $\mathrm{mg} / \mathrm{kg}$, i.r.) alone or co-administered with ivermectin (IVM, $0.2 \mathrm{mg} / \mathrm{kg}$, s.c.) and methimazole (MTZ, $1.5 \mathrm{mg} / \mathrm{kg}, \mathrm{i} . \mathrm{m}$.) to Fasciola hepatica-infected sheep.

\begin{tabular}{ccccc}
\hline PHARMACOKINETIC PARAMETERS & \multicolumn{2}{c}{ TCBZ.SO } & \multicolumn{2}{c}{ TCBZ.SO $_{2}$} \\
\cline { 2 - 5 } & TCBZ alone & Combined treatment & TCBZ alone & Combined treatment \\
\hline Cmax $(\mu \mathrm{g} / \mathrm{ml})$ & $14.0 \pm 0.85$ & $15.6 \pm 1.46$ & $13.5 \pm 1.68$ & $12.3 \pm 1.28$ \\
$\operatorname{Tmax}(\mathrm{h})$ & $22.5 \pm 7.55$ & $24.0 \pm 4.90$ & $39.0 \pm 6.00$ & $42.0 \pm 6.93$ \\
$\mathrm{AUC}_{0-\mathrm{t}}(\mu \mathrm{g} . \mathrm{h} / \mathrm{ml})$ & $653.9 \pm 140.6$ & $650.7 \pm 122.8$ & $868.2 \pm 217.6$ & $893.7 \pm 114.1$ \\
$\mathrm{AUC}_{0-\infty}(\mu \mathrm{g} . \mathrm{h} / \mathrm{ml})$ & $661.5 \pm 148.5$ & $657.1 \pm 119.4$ & $917.9 \pm 270.6$ & $945.6 \pm 129.3$ \\
$\mathrm{~T} 1 / 2 \mathrm{el}(\mathrm{h})$ & $17.5 \pm 8.45$ & $18.4 \pm 5.82$ & $26.8 \pm 10.9$ & $30.4 \pm 9.30$ \\
MRT $(\mathrm{h})$ & $38.8 \pm 10.5$ & $39.1 \pm 5.03$ & $61.3 \pm 17.2$ & $67.6 \pm 9.71$ \\
$\mathrm{~T} 1 / 2 \mathrm{for}(\mathrm{h})$ & $6.85 \pm 2.18$ & $8.26 \pm 1.22$ & $12.3 \pm 2.90$ & $13.5 \pm 1.93$ \\
\hline
\end{tabular}

Cmax: peak plasma concentration; Tmax: time to the $\mathrm{Cmax}_{1} \mathrm{AUC}_{0-\mathrm{t}}$ : Area under the plasma concentration vs. time curve from 0 to the detection time; $A \cup C_{0-\infty}(\mu \mathrm{g}$. $\mathrm{h} / \mathrm{ml}$ ): Area under the plasma concentration vs. time curve extrapolated to infinity; T1/2el: elimination half-life; MRT: mean residence time (obtained by noncompartmental analysis of the data); $\mathrm{T} 1 / 2$ for: formation half life. 
Table 2 Individual and mean fluke counts and clinical efficacy (\%) against triclabendazole (TCBZ)-resistant Fasciola hepatica obtained after the administration of TCBZ alone (10 $\mathrm{mg} / \mathrm{kg}$, i.r.) or co-administered with ivermectin (IVM, $0.2 \mathrm{mg} / \mathbf{k g}$, s.c.) and methimazole (MTZ, $1.5 \mathrm{mg} / \mathrm{kg}$, i.m.) to Fasciola hepatica-infected sheep.

\begin{tabular}{cccc}
\hline & $\begin{array}{c}\text { Untreated } \\
\text { control }\end{array}$ & $\begin{array}{c}\text { TCBZ } \\
\text { alone }\end{array}$ & $\begin{array}{c}\text { Combined } \\
\text { treatment }\end{array}$ \\
\hline 19 & 2 & 6 \\
& 11 & 12 & 9 \\
& 14 & 8 & 12 \\
& 9 & 5 & 6 \\
\hline Arithmetic & 13.25 & 6.75 & 8.25 \\
mean & - & $56 \%$ & $38 \%$ \\
Efficacy* & & & \\
\hline
\end{tabular}

* The efficacy was calculated using geometric means.

efficacy of TCBZ against TCBZ-resistant $F$. hepatica compared to the administration of TCBZ alone. This result may have two potential explanations: a) an alternative mechanism of TCBZ resistance may play a critical role under in vivo conditions, or b) the interaction between TCBZ-IVM-MTZ under our in vivo conditions does not achieve adequate magnitude at the level of the fluke to reverse TCBZ resistance. For example, the IVM concentration $(1 \mu \mathrm{g} / \mathrm{ml})$ used in the ex vivo experiments [7], is not achieved in bile after the s.c. administration of IVM $(0.2 \mathrm{mg} / \mathrm{kg})$ in sheep.

\section{Conclusions}

In conclusion, the presence of IVM and MTZ did not affect the disposition kinetics of TCBZ and its metabolites. Thus, the combined drug treatment did not reverse the poor efficacy of TCBZ against TCBZ-resistant $F$. hepatica.

\section{Acknowledgements}

The authors would like to acknowledge Dr. Gottfried Büscher, from Novartis Animal Health Inc., Basel, Switzerland, who kindly provided TCBZ and TCBZ. $\mathrm{SO}$ and $\mathrm{TCBZ} . \mathrm{SO}_{2}$ pure reference standards. This research was supported by the Agencia Nacional de Promoción Científica y Tecnológica, (Argentina).

\section{Author details \\ ${ }^{1}$ Laboratorio de Farmacología, Facultad de Ciencias Veterinarias, Universidad Nacional del Centro de la Provincia de Buenos Aires (UNCPBA), Campus Universitario, 7000, Tandil, Argentina . ${ }^{2}$ Consejo Nacional de Investigaciones Científicas y Técnicas (CONICET), Argentina. ${ }^{3}$ School of Biological Sciences, Medical Biology Centre, The Queen's University of Belfast, Belfast, Northern Ireland, BT9 7BL, UK.}

\section{Authors' contributions}

LM and LC participate in the animal and analytical phase of the experiment and in writing the draft manuscript. $L A$ and $C L$ conceived the study, participated in its design and in the animal phase, and revised the draft version of the manuscript. LS and IF produced the metacercaries and revised the draft version of the manuscript. All authors have read and approved the final manuscript.

\section{Competing interests}

The authors declare that they have no competing interests.

\section{Received: 27 August 2009}

Accepted: 3 February 2010 Published: 3 February 2010

\section{References}

1. Boray J, Crowfoot P, Strong M, Allison J, Schellenbaum M, von Orelli M, Sarasin G: Treatment of immature and mature Fasciola hepatica infections in sheep with triclabendazole. Vet Rec 1983, 113:315-317.

2. Fairweather I: Triclabendazole: new skills to unravel an old(ish) enigma. J Helmintol 2005, 79:227-234.

3. Fairweather I: Triclabendazole progress report, 2005-2009: an advancement of learning?. J Helminthol 2009, 83:139-150.

4. Ouellette M: Biochemical and molecular mechanism of drug resistance in parasites. Trop Med Int Health 2001, 6:874-882.

5. Robinson M, Lawson J, Trudgett A, Hoey E, Fairweather I: The comparative metabolism of triclabendazole sulphoxide by triclabendazole-susceptible and triclabendazole-resistant Fasciola hepatica. Parasitol Res 2004, 92:205-210.

6. Alvarez L, Solana H, Mottier L, Virkel G, Fairweather I, Lanusse C: Altered drug influx/efflux and enhanced metabolic activity in triclabendazoleresistant liver flukes. Parasitology 2005, 131:501-510.

7. Mottier L, Alvarez L, Fairweather I, Lanusse C: Resistance-induced changes in triclabendazole transport in Fasciola hepatica : Ivermectin reversal effect. J Parasitol 2006, 92:1355-1360.

8. Dupuy J, Lespine A, Alvinerie M: Influence of anthelmintic drugs on Pglycoprotein transport activity in mdr-1-LLC-PK1 cells. J Vet Pharmacol Therap 2006, 29:115-119.

9. Pouliot J, Lheureux F, Liu Z, Prichard R, Georges E: Reversal of Pglycoprotein-associated multidrug resistance by ivermectin. Biochem Pharmacol 1997, 53:17-25.

10. Xu M, Molento M, Blackhall W, Ribeiro $P$, Beech $P$, Prichard R: Ivermectin resistance in nematodes may be caused by alteration of $P$-glycoprotein homolog. Mol Biochem Parasitol 1998, 91:327-335.

11. Kerboeuf D, Blackhall W, Kaminsky R, Von Samson-Himmelstjerna G: Pglycoprotein in helminths: Function and perspectives for anthelmintic treatment and reversal of resistance. Int J Antimicrob Agents 2003, 22:332-346.

12. Wolstenholme A, Fairweather I, Prichard R, Von Samson-Himmelstjerna G, Sangster N: Drug resistance in veterinary parasites. Trends in Parasitol 2004, 20:469-476.

13. Didier A, Loor F: The abamectin derivative ivermectin is a potent $\mathbf{P}$ glycoprotein inhibitor. Anticancer Drugs 1996, 7:745-751.

14. Halferty L, Brennan GP, Trudgett A, Hoey L, Fairweather I: Relative activity of triclabendazole metabolites against the liver fluke, Fasciola hepatica. Veterinary Parasitology 159:126-138.

15. Devine C, Brennan GP, Lanusse C, Alvarez L, Trudgett A, Hoey E, Fairweather I: Effect of the metabolic inhibitor, methimazole on the drug susceptibility of a triclabendazole-resistant isolate of Fasciola hepatica. Parasitology 2009, 136:183-192.

16. Alvarez L, Lifschitz A, Entrocasso C, Manazza J, Mottier L, Borda B, Virkel G, Lanusse $C$ : Evaluation of the interaction between ivermectin and albendazole following their combined use in lambs. J Vet Pharmacol Therap 2008, 31:230-239.

17. Lifschitz A, Virkel G, Ballent M, Sallovitz J, Lanusse C: Combined use of ivermectin and triclabendazole in sheep: in vitro and in vivo characterisation of their pharmacological interaction. Vet J 2009, 182:261-268.

18. Virkel G, Lifschitz A, Sallovitz J, Pis A, Lanusse C: Assessment of the main metabolism pathways for the flukicidal compound triclabendazole in sheep. J Vet Pharmacol Therap 2006, 29:213-223.

19. Virkel G, Lifschitz A, Sallovitz J, Ballent M, Scarcella S, Lanusse C: Inhibition of cytochrome P450 activity enhances the systemic availability of triclabendazole metabolites in sheep. J Vet Pharmacol Therap 2009, 32:79-86.

20. Ministry of Agriculture Fisheries and Food (M.A.F.F.): Manual of Veterinary Parasitological Laboratory Techniques London, England 1987.

21. AVMA: Report of the AVMA panel on euthanasia. J Am Vet Med Assoc 2001, 218:669-696 
22. Coles GC, Stafford KA: Activity of oxyclozanide, nitroxynil, clorsulon and albendazole against adult triclabendazole-resistant Fasciola hepatica. Vet Rec 2001, 148:723-724.

23. McConville M, Brennan GP, Flanagan A, Edgar HWJ, Hanna REB, McCoy M, Gordon AW, Castillo R, Hernández-Campos A, Fairweather I: An evaluation of the efficacy of compound alpha and triclabendazole against two isolates of Fasciola hepatica. Vet Parasitol 2009, 162:75-88.

24. Lanusse C, Prichard R: Methimazole increases the plasma concentration of the albendazole metabolites of netobimin in sheep. Biopharm Drug Dispos 1992, 13:95-103.

25. Wood IB, Amaral NK, Bairden K, Duncan JL, Kassai T, Malone JB, Pankavich JA, Reinecke RK, Slocombe O, Taylor SM, Vercruysse J: World Association for the Advancement of Veterinary Parasitology (W.A.A.V.P.) second edition of guidelines for evaluating the efficacy of anthelmintics in ruminants (bovine, ovine, caprine). Vet Parasitol 1995, 58:181-213.

26. Gibaldi M, Perrier D: Pharmacokinetics New York, Marcel Dekker 1982.

27. Hennessy D, Lacey E, Steel J, Prichard R: The kinetics of triclabendazole disposition in sheep. J Vet Pharmacol Therap 1987, 10:64-72.

28. Lanusse C, Gascon L, Prichard R: Comparative plasma disposition kinetics of albendazole, fenbendazole, oxfendazole and their metabolites in adult sheep. J Vet Pharmacol Therap 1995, 18:196-203.

29. Lanusse C, Gascon L, Prichard R: Influence of the antithyroid compound methimazole on the plasma disposition of fenbendazole and oxfendazole in sheep. Res Vet Sci 1995, 58:222-226.

30. Lanusse C, Prichard R: Enhancement of the plasma concentration of albendazole sulphoxide in sheep following coadministration of parenteral netobimin and liver oxidase inhibitors. Res Vet Sci 1991, 51:306-312.

31. Klotz U, Ogbuokiri J, Okonkwo P: Ivermectin binds avidly to plasma proteins. Eur J Clin Pharmacol 1990, 39:607-608.

32. McCoy MA, Fairweather I, Brennan GP, Kenny JM, Ellison S, Forbes AB: The efficacy of nitroxynil and triclabendazole administered synchronously against juvenile triclabendazole-resistant Fasciola hepatica in sheep. Res Vet Sci 2005, 78(Suppl A):33.

doi:10.1186/1746-6148-6-8

Cite this article as: Ceballos et al:: Unchanged triclabendazole kinetics after co-administration with ivermectin and methimazole: failure of its therapeutic activity against triclabendazole-resistant liver flukes. BMC

Veterinary Research 2010 6:8.

\section{Submit your next manuscript to BioMed Central and take full advantage of:}

- Convenient online submission

- Thorough peer review

- No space constraints or color figure charges

- Immediate publication on acceptance

- Inclusion in PubMed, CAS, Scopus and Google Scholar

- Research which is freely available for redistribution 